\title{
INDUCTION DE LA MATURATION OVOCYTAIRE ET DE L'OVULATION PAR TRAITEMENT HORMONAL CHEZ LE BROCHET (ESOX LUCIUS)
}

\author{
C. BRY, R. BILLARD, G. de MONTALEMBERT \\ Laboratoire de Physiologie des Poissons, I.NR.A \\ 78350 JOUY-EN-JOSAS, France
}

\section{RESUME}

Une expérimentation a été conduite en vue de mettre au point une technique d'induction de la maturation ovocytaire et de l'ovulation par traitement hormonal chez le Brochet Esox lucius. Les résultats obtenus montrent :

1) La possibilite d'induire la maturation ovocytaire et l'ovulation par une seule injection intrapéritonéale d'hormone gonadotrope de saumon partiellement purifiée $(0.1 \mathrm{mg} / \mathrm{kg})$ effectuée dans les 24 heures qui suivent la capture.

Le taux d'ovulation est élevé : $89 \%$ en moyenne, et les ovules obtenus sont de bonne qualité (taux de fécondation estimé à 40 degrés-jours : $83 \%$ en moyenne).

A $12 \circ \mathrm{C}$, l'ovulation intervient 4 jours après l'injection hormonale. L'insémination artificielle peut a'ors s'opérer sur les ovules des différentes femelles que le traitement a synchronisèes. Les ovules doivent être inséminés dans les 24 heures, sous peine de vieillissement et de chute rapide de la fécondabilité. Une bonne estimation du bilan final de l'incubation peut être obtenue par simple dénombrement des "œufs blancs " à 40 degrés-jours.

2) La dégénérescence rapide d'une grande proportion d'ovocytes consécutive au transport des femelles et à leur maintien en captivité : le taux moyen d'ovulation, de $96 \%$ si le traitement est appliqué immediatement après la capture, chute à $40 \%$ si l'animal est traité après 3 jours de captivité.

Article available at http://www.kmae-journal.org or http://dx.doi.org/10.1051/kmae:1978003 


\section{INTRODUCTION}

Une production régulière et quantitative de brochetons ne peut s'envisager qu'avec la possibilité d'obtenir, en nombre suffisant, des géniteurs femelles matures. Or, l'ovulation ne se produit généralement pas de façon spontanée chez des femelles Brochet capturées à l'époque de la reproduction et maintenues dans des conditions de confinement. De plus, la plupart des femelles, alors en fin de vitellogenèse, présentent, après capture, des phénomènes de dégénérescence au niveau des ovaires, et des mortalités importantes surviennent au bout de quelques semaines de captivité (PORTAL, 1947; CHIMITS, 1947 et 1956). Lorsque le stockage des géniteurs s'effectue en petits étangs, les taux de survie sont généralement satisfaisants et la séquence maturation ovocytaire-ovulation est susceptible de se dérouler normalement. suivie de l'oviposition et de la fécondation (SCHAPERCLAUS, 1961; KAUSCH, 1976). Le rendement d'une telle opération (nombre d'alevins nageants oul de brochetons récoltés/nombre potentiel d'œufs) a été rarement évalué et semble faible (inférieur à $1 \%$ - ROYER, 1971), probablement du même ordre çue celui observé sur les aires de fraie naturelles (moins de 0,5\% - CARBINE, 1944).

En se basant sur l'observation du comportement de fraie ou en contrôlant les femelles tous les deux jours, il est possible de prélever des femelles peu après l'ovulation (HUET, 1972, 1976).

Cette technique de maturation des géniteurs femelles en petits étangs s'avère cependant difficilement généralisable. En effet, les phases délicates de la maturation ovocytaire et de l'ovulation restent soumises aux aléas climatiques, en particulier à la possibilité de fluctuations importantes de la température (HUET, 1972). D'autre part, les ovulations sont rarement synchronisées et peuvent s'échelonner, d'où l'obtention d'alevins d'âges différents qu'il faudra séparer en autant de lots pour la période de grossissement. C'est pourquoi nous avons envisagé au laboratoire une approche différente, en expérimentant un certain nombre de traitements hormonaux susceptibles d'induire l'ovulation de femelles après capture. La technique dite "d'hypophysation" qui consiste à administrer à un poisson un homogénat ou un extrait plus ou moins purifié d'hypophyses a déjà été appliquée au Brochet (SORENSON et al., 1966), mais le stade de maturité des femelles avant traitement. ainsi que le rendement de l'opération. n'ont pas été précisés. Dans la présente expérimentation, nous avons tenté de réduire la quantité de préparation hypophysaire injectée par adjonction d'un progestagène (17x-hydroxy-203-dihydroprogestérone : $17 x-203 \mathrm{P})$. Ce progestagène s'est en effet révélé capable d'induire la maturation d'ovocytes de brochet in vitro (JALABERT et BRETON, 1973). D'autre part, cette association d'un extrait hypophysaire et du même progestagène s'est avérée efficace chez la Carpe dans certaines conditions de temperature (JALABERT et al, 1977).

Des résultats partiels concernant ce travail ont déjà èté rapportés (de MONTALEMBERT et al., 1978 a et b).

\section{MATERIEL ET METHODES}

Les expérimentations se sont déroulées au laboratoire, à Jouy-en-Josas, au printemps 1977. Des femelles de 2 à 3 ans, pesant entre $800 \mathrm{~g}$ et $2 \mathrm{~kg}$, provenaient d'étangs des Dombes, après avoir transité quelques heures dans des viviers flottants établis sur le Rhône (température de l'eau : de 9 à $10 \circ \mathrm{C}$ ). Le stockage des femelles au laboratoire était assuré à l'obscurité en eau recyclée d'une température d'environ $12^{\circ} \mathrm{C}$ (PETIT, 1973). Les animaux ètaient anesthésiés au Phénoxyéthanol $(0,5 \mathrm{~m} / / 1)$ avant chaque intervention. 


\section{Matériel injecté}

Les injections ont été pratiquées dans la cavité générale, l'aiguille (Becton Dickinson, no $21 \mathrm{G1} / 2$ ) ètant implantée sous l'une des nageoires pelviennes. Chaque femelle reçoit un volume total de $1 \mathrm{ml} / \mathrm{kg}$. Les préparations hormonales injectées sont les suivantes et ont été detaillèes précédemment (de MONTALEMBERT et al., 1978 a) :

- Gonadotropine de saumon partiellement purifièe (GSPP):

- 17x-203 P : cette hormone steroide est tout d'abord dissoute dans de l'alcool (solution mere), puis mise en suspension dans du liquide physiologique $(\mathrm{NaCl}$ $8 \%$ ) juste avant l'injection (taux d'alcool dans le produit injecté : 20\%);

- HCG.

\section{Détermination des stades ovocytaires}

L'évolution de la maturation ovocytaire a été suvie, avant et après traitement hormonal, grâce à des prélèvements d'ovocytes (légère pression abdominale et expulsion de quelques ovocytes par l'orifice génital). Après immersion dans la solution de Stockard, destinè à rendre visible la vésicule germinale, les stades de maturation ovocytaire sont définis selon la classification suivante (JALABERT et al., 1976) :

- $\quad \mathrm{i}$ : vésicule germinale non périphérique ;

- 1: vésicule germinale périphérique ;

- 2: ovocyte en cours de maturation (vésicule germinale encore visible);

- 3: ovocyte maturé (après rupture de la vésicule);

- 4: ovocyte maturé et ovulé

En début d'expérience, toutes les femelles étaient au stade $i$.

\section{Détermination du taux d'ovulation}

Les femelles ovulees ont été sacrifiées immédiatement après prélèvement des ovules et les femelles non ovulées 8 jours après l'injection hormonale.

Les ovules recueillis ou restant dans le tractus génital femelle (la somme des deux représentant la quantité d'ovocytes ovulés), ainsi que les reliquats ovariens, ont été pesés dans tous les cas et le pourcentage d'ovulation (PO) est érabli comme suit :

$\mathrm{PO}=$ poids des ovocytes ovules / poids initial de l'ovaire (poids initial de l'ovaire $=$ poids des reliquats ovariens + poids des ovocytes ovulés).

\section{Détermination du taux de fécondation}

Le taux de fécondation des ovules a été estimé d'après le pourcentage d'œufs embryonnés à 40 degrés-jours. Pour cela, environ 300 ovules par femelle sont inséminés clans $10 \mathrm{ml}$ de dilueur 532 (BILLARD, 1977) avec $10 \mu /$ de sperme provenant d'un pool de 10 mâles et mis en incubation à $12{ }^{\circ} \mathrm{C}$ sur grillage fin.

\section{Analyse statistique}

La comparaison entre les divers traitements a èté faite avec le test $F$, après transformation angulaire des valeurs des pourcentages. L'intervalle de confiance à $95 \%$ est porté sur les graphiques et le tableau 


\section{Expériences réalisées}

\section{- Efficacité de diverses préparations hormonales sur l'ovulation}

Les préparations hormonales mentionnées ci-dessus ont été administrées lors de la capture, seules ou en association séquentielle à 24 heures d'intervalle, à des lots de femelles (n variant de 7 à 15) :

GSPP : 0,$1 ; 0,03 \mathrm{mg} / \mathrm{kg}$ de poids vif;

HCG : $2000 \mathrm{UI} / \mathrm{kg}$;

$17 x-20 \beta \mathrm{P}: 3 \mathrm{mg} / \mathrm{kg}$;

$\operatorname{GSPP}(0,03 \mathrm{mg} / \mathrm{kg})+17 x-20 \beta \mathrm{P}(3 \mathrm{mg} / \mathrm{kg})$.

- Effet du transport des gèniteurs et de leur maintien en captivité sur l'efficacité du traitement hormonal

Dans cette expérience, les injections hormonales (GSPP $0.1 \mathrm{mg} / \mathrm{kg}$ ) ont été faites à des temps croissants après la capture. Les injections ont été pratiquées lors de la capture et 1 ou 3 jours après. Le taux d'ovulation et la fécondabilité des ovules ont été mesurés.

\section{- Effet de la rétention des ovules sur leur fécondabilité}

La rétention des ovules dans le tractus génital femelle après ovulation et ses effets sur leur aptitude à être fécondés a été testée. Deux types d'expériences ont été pratiquées. Dans la premiere (A), 5 groupes de 2 femelles ont été utilisés. Pour chaque femelle l'ovulation a été induite par GSPP $(0,1 \mathrm{mg} / \mathrm{kg})$ et 1000 ovules ont été inséminés et mis en incubation selon la procédure décrite ci-dessus. Ces opérations de prélèvement d'ovules et d'insémination ont été pratiquées à des temps croissants après ovulation : le jour même de l'ovulation (Jo) pour le groupe 1, un jour après ovulation pour le groupe 2 et ainsi de suite jusqu'à 4 jours. Les taux de fécondation ont été évalués à 40 degrés-jours. Dans une seconde expérience (B), 7 femelles, maintenues à une température constante de $13 \circ \mathrm{C}$, ont reçu un traitement séquentiel de préparation gonadotrope et de $17 \alpha-20 \beta$ P $(3 \mathrm{mg} / \mathrm{kg}) 24$ heures pius tard; sur chaque femelle, 400 ovules ont été prélevés, le jour de l'ovulation et au cours des 5 jours suivants, et inséminés comme décrit ci-dessus en vue d'établir le pourcentage d'éclosion.

\section{- Appréciation du taux d'ovules non fécondés et de l'évolution du taux de mortalité embryonnaire par comptage des ceufs blancs}

Ces observations complémentaires ont été conduites afin de déterminer si l'évaluation du taux d'œufs embryonnès à 40 degrés-jours constituait un critère fidèle d'appréciation du rendement global de i'incubation (taux d'éclosion).

5 lots de 200 ovules ont èté prélevés sur 5 femelles, moins de 24 heures après ovulation induite, inséminés et incubès sur clayettes à température constante $(13 \circ \mathrm{C})$ selon le protocole déjà décrit.

Les œufs blancs ont été dénombrés chaque jour jusqu'à l'èclosion. 


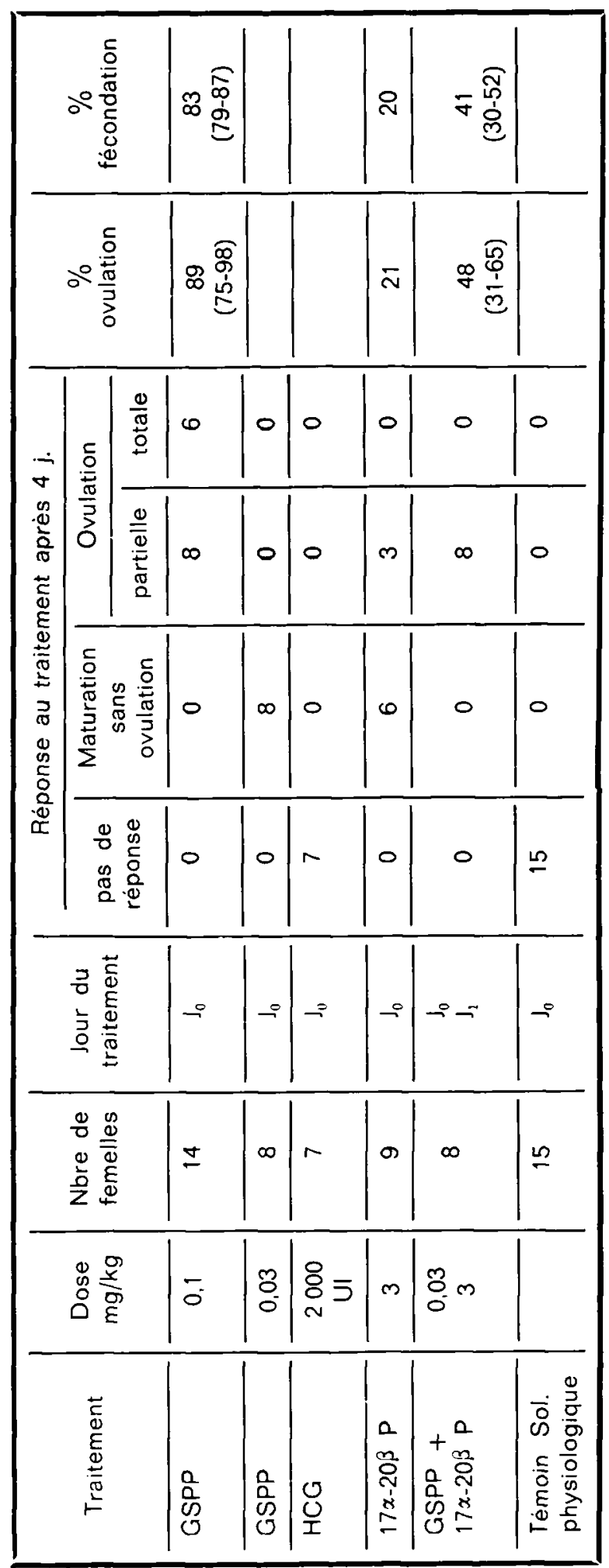

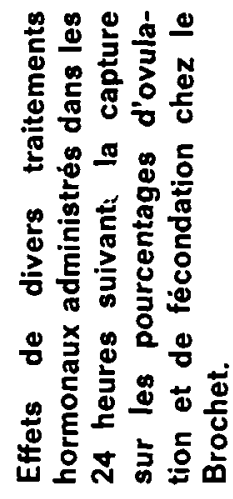

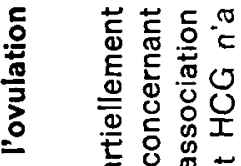
㐘 80

声

-

\%

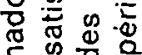

吕必串

으 票

0.

心

$\omega$

可

क 음

总完蓄

为

先 $\mathrm{E}$ 음

- $\quad \stackrel{0}{0} \overline{0}$ के

$\stackrel{\Phi}{=} \stackrel{\Phi}{\mathbb{N}}_{0}$

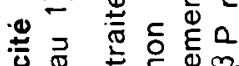

\&

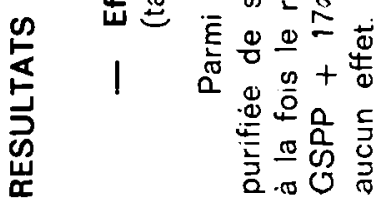

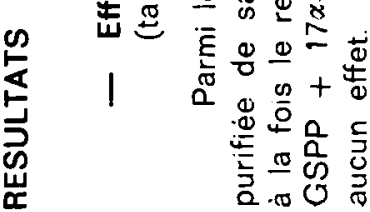



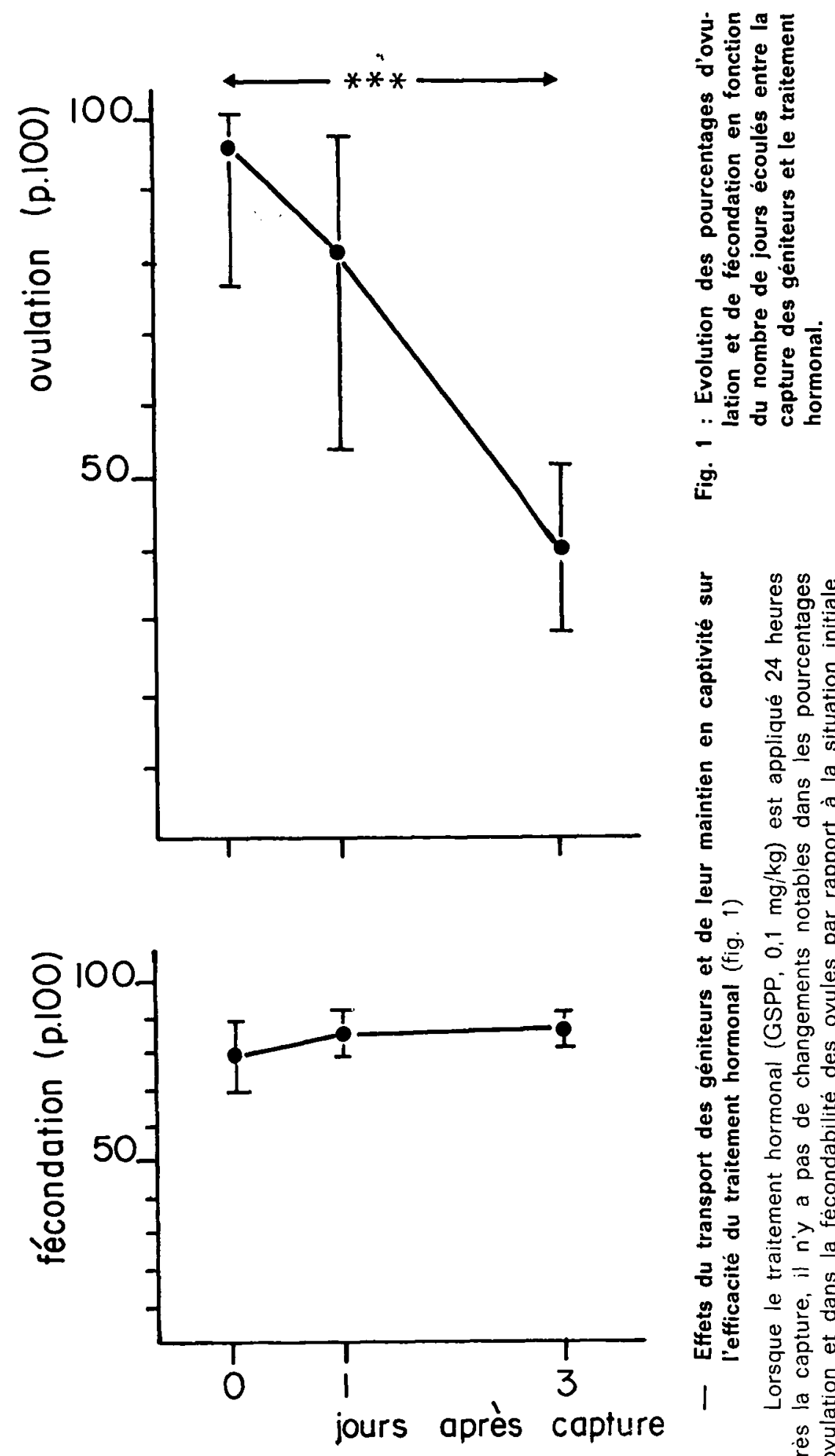

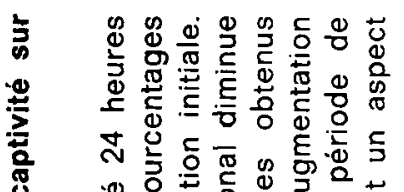

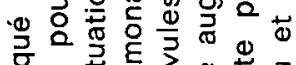

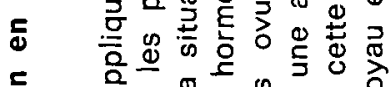

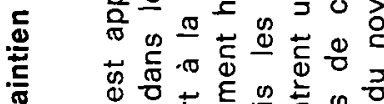

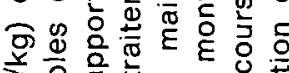

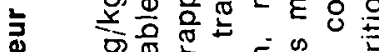

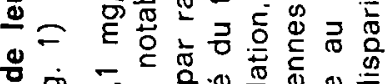

ष

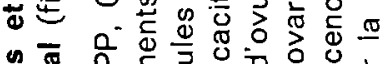

宩要

壳

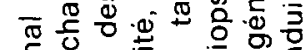

등

范

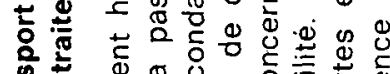

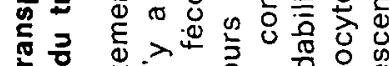

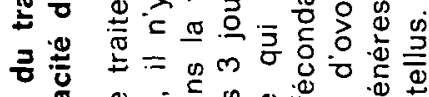

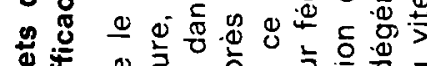

昰昰

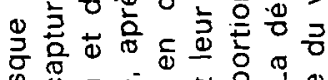
कั

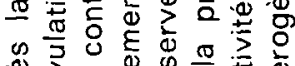

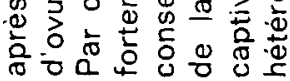


- Effet de la rétention des ovules sur leur fécondabilité (fig. 2)

Les effets de la rétention des ovules dans le tractus génital femelle pendant des temps croissants ont été appréciés par l'évolution du pourcentage de fécondation dans le cas de l'expérience A (fig. 2 A). Une diminution significative $(P<0,001)$ de la fécondabilité des ovules a été observée entre le $2^{\theta}$ et le $3^{e}$ jour de rétention. Dans l'expérience $B$ où l'incubation a été conduite à son terme et les résultats représentés par le pourcentage d'éclosion, une altération significative est observée dès le $2^{e}$ jour $(P<0,05)$ et la diminution du taux d'éclosion est encore plus marquèe entre le $2^{e}$ et le $3^{e}$ jour de rétention.

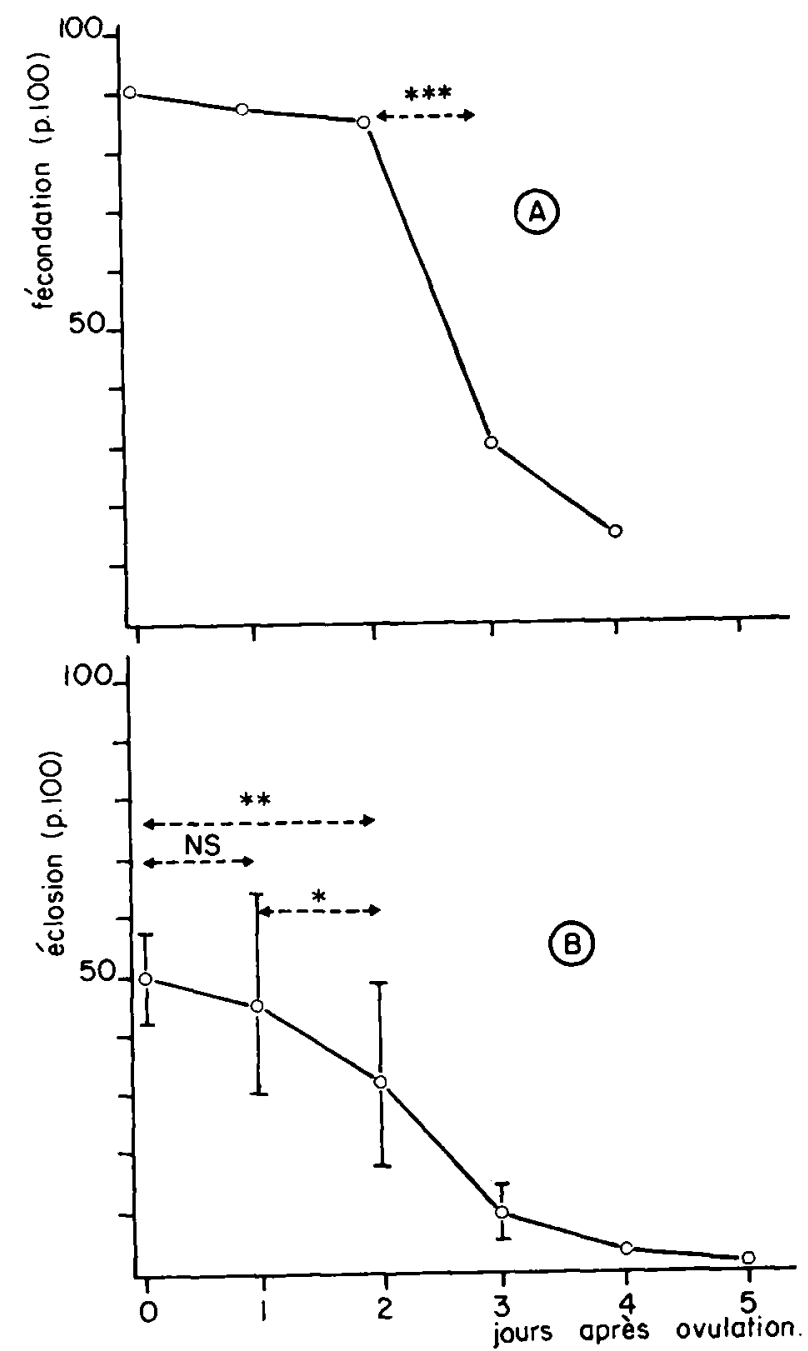

Fig. 2 : Evolution de la qualité des ovules après rétention dans le tractus génital femelle pendant des temps croissants après ovulation.

A. La totalité des ovules a été prélevée sur 5 groupes de 2 femelles, entre 0 et 4 jours après ovulation. Le pourcentage d'œufs embryonnés a été déterminé à $\mathbf{4 0}$ degrés-jours.

B. Des ovules ont été prélevés chaque jour sur 7 femelles pendant une période de 5 jours après ovulation.

Le bilan de l'incubation a été fait à l'éclosion. 


\section{- Evolution du taux de mortalité embryonnaire (fig. 3)}

Les pourcentages cumulés d'œufs blancs montrent que 80 à $90 \%$ de la totalité des œufs blancs enregistrés au cours de l'incubation sont identifiables au cours des trois premiers jours et en particulier le 3 e jour, c'est-à-dire, pour une température constante de $13 \circ \mathrm{C}$, avant 40 degrés-jours. On peut donc avoir une bonne estimation du rendement global de l'incubation par simple dénombrement des œufs blancs à 40 degrés-jours.

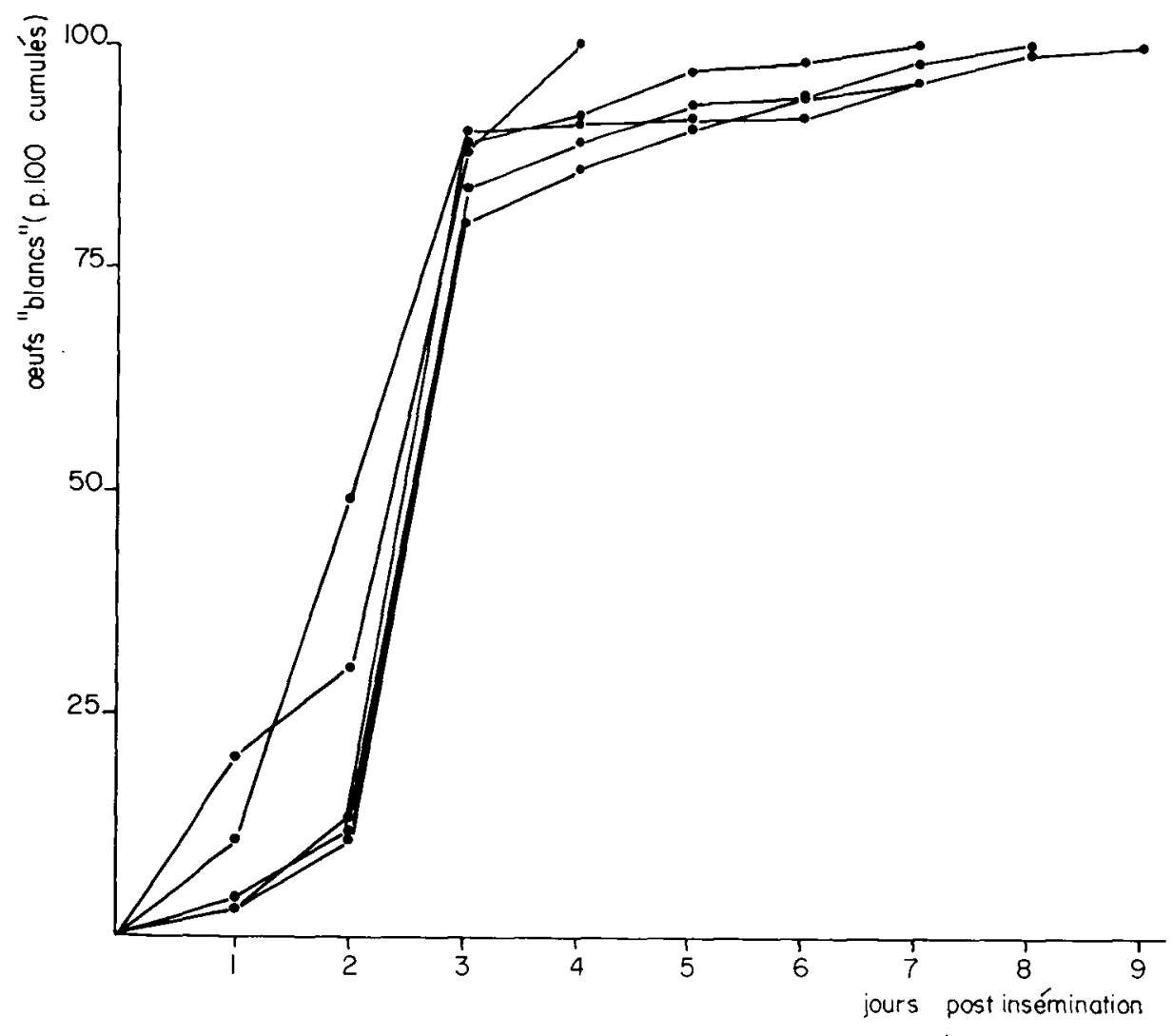

Fig. 3 : Evolution de l'apparition des œufs blancs (ovules non fécondés et œufs abortifs), exprimée en pourcentages cumulés, au cours de l'incubation. Temperature d'incubation : $13 \circ \mathrm{C}$. 


\section{DISCUSSION}

Ces résultats montrent qu'une seule injection d'extrait d'hypophyses de saumon, administree en fin de vitellogerièse et avant achèvement de la migration de la vésicule germinale, peut induire l'ovulation de Brochets femelles. Cet extrait hypophysaire est d'activité biologique connue : l'activité gonadotrope mesurée par le test de maturation in vitro d'ovocytes de truite (JALABERT et al., 1974) est légèrement supérieure à celle d'une autre préparation d'hypophyses de saumon SG-G100 (DONALDSON et al., 1972) et 5,7 fois moins active que l'hormone gonadotrope de saumon hautement purifiee (JALABERT et BRETON, non publié). En outre, la préparation de GSPP est standardisée et basée sur la méthode décrite par IDLER et al. (1975) et modifiee par BRETON et al. (1978). Les doses administrées doivent être connues avec précision. En effet, la dose de $0,03 \mathrm{mg} / \mathrm{kg}$ provoque la maturation, mais pas l'ovulation alors qu'une dose trois plus élevee provoque à la fois maturation et ovulation. De ce fait, l'emploi de broyats hypophysaires est à proscire car l'activité gonadotrope d'une telle préparation n'est pas connue et peut varier d'un facteur 3 et davantage en fonction de l'origine des donneurs et de l'époque du prélèvement (BRETON et BILLARD, non publié). En outre, un homogénat hypophysaire implique un apport élevé de protéines étrangères qui est susceptible de provoquer une perturbation supplèmentaire. Les conséquences du traitement avec GSPP à $0,1 \mathrm{mg} / \mathrm{kg}$ sur les cycles reproducteurs ultérieurs n'ont pas été recherchées et restent à étudier. Les ovules obtenus par ce traitement sont de bonne qualité comme le montrent les pourcentages de fécondation estimès à 40 degrés-jours ( $83 \%$ en moyenne).

Cependant, le même traitement $(0,1 \mathrm{mg}$ GSPP $/ \mathrm{kg})$ appliqué en mars 1978 à cinq femelles dès leur capture dans la région de la Somme a donné des taux d'ovulation satisfaisants, mais des fécondabilités inférieures et plus hétérogènes ( $45 \%$ en moyenne, valeurs extrêmes : 13 et $84 \%$ ). II semble, par ailleurs, que les femelles qui ovulent spontanement dans la nature fournissent également des ovules d'une qualité très variable (WESTERS, communication personnelle). Une possible rétention des ovules ne peut être exclue dans ce dernier cas, mais les conditions climatiques (en particulier le profil thermique au cours de la vitellogenèse), l'alimentation et des facteurs propres à chaque femelle méritent d'être étudiés comme causes possibles de cette variabilité.

Dans la plupart des expériences, la survie embryonnaire au-delà de 40 degrés-jours n'a pas été étudiée. Cependant, d'après l'expérience rapportee dans la figure 3, le pourcentage d'œufs embryonnés à 40 degrés-jours (déterminé par examen des embryons ou par dénombrement des a œufs blancs *) donne une bonne estimation du taux d'éclosion puisque la majorité des œufs abortifs ou non fécondés est identifiable dès le troisième jour d'incubation $(a ̀ 13 \circ \mathrm{C})$. Cette conclusion reste cependant limitée aux conditions de la présente expérience : ovules fraichement ovulés au départ et incubations sur clayette. En effet, l'évolution du taux de mortalité est differente et peut intervenir au-delà de 40 degrés-jours dans le cas d'ovules "vieillis " dans lë tractus génital femelle après ovulation (BRY, non publié).

L'emploi de $17 x-20 \beta P$, seule ou en association avec GSPP à faible dose, ne donne que des ovulations partielles (taux d'ovulation inférieur à $50 \%$ ) et des fécondabilités médiocres $(20$ à $40 \%$ ), et ne peut être retenu on vue d'application pratique. II est possible que, comme dans le cas de la Truite arc-en-ciel (JALABERT et al., 1976, 1978), l'administration du traitement séquentiel GSPP $+17 \alpha-203 \mathrm{P}$ ou de $17 x-20 \hat{\mathrm{P}} \mathrm{P}$ seule à un stade plus avancé de la migration nucléaire (position subpériphérique) donne de meilleurs taux d'ovulation et de fécondation. 
HCG qui se révèle capable d'induire l'ovulation chez certains poissons téléostéens (SHEHADEH, 1975) n'a eu aucun effet chez le Brochet dans les conditions de la présente expérience.

La diminution de la réponse au traitement par GSPP chez les femelies maintenues en captivité pendant quelques jours constitue un fait extrêmement important. Les phénomènes de dégénerescence ovocytaire que nous avons observés résultent très vraisemblablement des conditions de manipulation et de confinement. II apparait donc que les conditions de stockage et de manipulation des géniteurs femelies en fin de vitellogenèse sont de nature à altérer leur capacité de reproduction. Il reste à définir si, à des stades plus précoces du développement des gonades, les animaux présentent la même susceptibilité aux manipulations (pendant la vitellogenèse par exemple).

La chute de fécondabilité des ovules laissés dans le tractus génital de la femelle apparait rapide chez le Brochet ( 2 à 3 jours) alors qu'elle n'intervient qu'après huit jours au moins chez la Truite arc-en-ciel (SAKAl et al., 1975; ESCAFFRE et al., 1976; BRY, non publié). Cette baisse de fécondabilité s'est produite au troisième jour dans l'expérience $A$ et au deuxième dans l'expérience $B$. II faut préciser que, dans ce dernier cas, la température d'élevage était légèrement plus élevée $\left(13^{\circ} \mathrm{C}\right.$ au lieu de $12 \circ \mathrm{C}$ dans lexpérience $\left.\mathrm{A}\right)$ et que les femelles avaient subi des manipulations répétées pendant six jours. En outre, le traitement était différent et faisait intervenir la $17 \alpha-20 \beta \mathrm{P}$ dans l'expérience $\mathrm{B}$. Ces différences peuvent expliquer la chute plus précoce de fertilité dans l'expérience $B$.

\section{REMERCIEMENTS}

Cette étude a été prise en charge par le Conseil Supérieur de la Pêche.

\section{SUMMARY}

The efficiency of various hormonal treatments (partially purified salmon gonadotropin - PPSG and HCG administered alone or in association with $17 x$ hydroxy-20 3 -dihydroprogesterone - $17 x-20 \beta \mathrm{P}$ ) on oocyte maturation and ovulation was investigated in submature northern pike (Esox lucius) females (at the initial oocyte stage, the germinal vesicle was in peripheral position).

A dose of $0.1 \mathrm{mg}$ PPSG $/ \mathrm{kg}$ body weight gave satisfactory ovulation ( $89 \%$ ) and fertilization $(83 \%)$ rates. Ovulation occured 4 days after treatment. A dose of $0.03 \mathrm{mg} / \mathrm{kg}$ induced oocyte maturation but not ovulation. A dose of $0.03 \mathrm{mg}$ $\mathrm{PPSG} / \mathrm{kg}$ followed 24 hours later by $3 \mathrm{mg} 17 x-20 \beta \mathrm{P} / \mathrm{kg}$ induced full maturation and partial ovulation. A single dose of $3 \mathrm{mg} 17 x-20 ; \mathrm{P}$ caused oocyte maturation in all animals and partial ovulation or no ovulation. In all treatments including $0.03 \mathrm{mg}$ PPSG $/ \mathrm{kg}$ or less, fertilization rates were low (20 to $40 \%$ ).

A three day captivity prior to treatment led to ovarian atresia and to a significant decrease in ovulation rates. When eggs were left in the ovaries after ovulation, a significant drop of fertility due to aging occured within 2 to 3 days.

A good estimate of the overall incubation success can be provided by a simple count of dead eggs at 40 degree-days. 


\section{BIBLIOGRAPHIE}

BILLARD R., 1977. Utilisation d'un systeme Tris-glycocolle pour tamponner le dilueur d'insémination pour truite. Bull. Fr. Piscic., 264, 102-112.

BRETON B., PRUNET P., RAINAUD P., 1978. Sexual differences in fish gonadotropins. Ann. Biol. anim. Bioch. Biophys., 18, 759-765.

CARBINE W.F., 1944. Egg production of the northern pike, Esox lucius and the percentage of survival of eggs and young on the spawning grounds. Pap. Mich. Acad. Sci., 29, 123-137.

CHIMITS P., 1947. Note sur le repeuplement artificiel du brochet. Bull. Fr. Piscic., 146, $16-24$.

CHIMITS P., 1956. Le brochet. Bull. Fr. Piscic., 180, 81-96.

DONALDSON E.M., YAMAZAKI F., DYE H.M., PHILLEO W.W., 1972. Preparation of gonadotropin from Salmon (Oncorhynchus tshawytscha) pituitary glands. Gen. Comp. Endocrinol., 18, 469-481.

ESCAFFRE A.M., PETIT J., BILLARD R., 1976. Evolution de la fécondabilité des ovules de truite arc-en-cie! laissés dans la cavité cœlomique après ovulation. Ile Congrès Europ. Ichthyol., Paris, septembre 1976.

HUET M., 1972. Elevage et maturation de géniteurs brochets en petits étangs. Verh. Internat. Verein. Limnol., 18, 1 128-1 134.

HUET M. 1976. Reproduction, incubation et alevinage du brochet (Esox lucius). EIFAC (Eur. Inland Fish. Advis Comm.) Tech. Pap., 25, 147-163.

IDLER D.R., BAZAR L.S., HWANG S.J., 1975. Fish gonadotropin(s). II - Isolation of gonadotropin(s) from chum salmon pituitary glands using affinity chromatography. Endocr. Res. Commun., 2, 215-235.

JALABERT B., BRETON B., 1973. Maturation in vitro des ovocytes de brochet Esox lucius. $7^{\text {th }}$ Conference of European Comparative Endocrinologists, 2731 août, BUDAPEST 1973

JALABERT B., BRETON B, BILLARD R., 1974. Dosage biologique des hormones gonadotropes de poissons par le test de maturation in vitro des ovocytes de truite. Ann. Biol. anim. Bioch. Biophys., 14, 217-228.

JALABERT B., BRY C., BRETON B., CAMPBELL C., 1976. Action de la 17xhydroxy-203-dihydroprogestérone et de la progestérone sur la maturation et l'ovulation in vivo et sur le niveau d'hormone gonadotrope plasmatique t-GTH chez la truite arc-en-ciel. C. R. Acad. Sci. Paris, série D, 283, 1 205-1 208.

JALABERT B., BRETON B., BRZUSJA E., FOSTIER A., WIENIAWSKI J., 1977. A new tool for induced spawning; the use of $17 x$-hydroxy-203-dihydroprogesterone to spawn carp at low temperature Aquaculture, 10, 353-364.

JALABERT B., BRETON B., FOSTIER A., 1978. Precocious induction of oocyte maturation and ovulation in rainbow trout (Salmo gairdneri) : problems when using 17x-hydroxy-203-dihydroprogesterone. Ann. Biol. anim. Bioch. Biophys., 18 (4), 977-984.

KAUSCH H., 1976. Breeding habits of the major cultivated fishes of EIFAC regions and problems of sexual maturation in captivity. In : Workshop on controlled reproduction of cultivated fishes. EIFAC Tech. Pap., 25, 43-52.

De MONTALEMBERT G., JALABERT B., BRY C., 1978 a. Precocious induction of maturation and ovulation in northern pike (Esox lucius). Ann. Biol anim. Bioch. Biophys., 18 (4), 969-975. 
De MONTALEMBERT G. BRY C., BILLARD R., $1978 \mathrm{~b}$. Control of reproduction in northern pike (Esox lucius). Trans. Amer. Fish. Soc. (in press)

PETIT J., 1973. Recyclage et épuration en pisciculture : étude theorique et bibliographique. Coll. Aqua CNEXO, Brest. octobre 1973, 445-470.

PORTAL J., 1947. Observations sur la pisciculture artificielle du brochet. Bull. Fr. Piscic., 147, 61-70.

ROYER L.M., 1971. Comparative production of Pike fingerlings from adult spewners and from fry planted in a controlled spawning marsh. Prog. Fish. Cult., 33, 153-155.

SAKAI K., NOMURA M., TAKASHIMA $F$., OTO $H_{\text {., }}$ 1975. The over-ripening phenomenon of rainbow trout. II - Changes in the percentage of eyed eggs, hatching rate and incidence of abnormal alevins during the process of over-ripening. Bull. Jap. Soc. Sci. Fish, 41, 855-860

SCHAPERCLAUS W., 1961. Lehrbuch der Teichwirtschaft. Berlin et Hambourg, Paul Parey, $2^{\text {te }}$ Auf.. $582 \mathrm{pp}$.

SHEHADEH Z.H, 1975. Induced breeding techniques. A review of progress and problems. In : Workshop on controlled reproduction of cultivated fishes. EIFAC Tech. Pap., 25, 72-89.

SORENSON L., BUSS K. BRADFORD A.D., 1966. The artificial propagation of esocid fishes in Pennsylvania. Prog. Fish. Cult., 28, 133-141. 